\title{
Diálisis peritoneal en tiempos de la enfermedad por coronavirus 2019: experiencia de cinco casos en un hospital de la Ciudad de México
}

\author{
Juan D. Díaz-García*, Joana Balderas-Juárez, Pamela Vargas-Solís, Joanger S. Cárdenas-Hurtado y \\ Brenda G. Delgado-Ávila \\ Servicio de Medicina Interna, Hospital General Dr. Manuel Gea González, Ciudad de México, México
}

\section{Resumen}

La lesión renal aguda se produce en el 4-23\% de los casos en pacientes con coronavirus 2 del síndrome respiratorio agudo grave (SARS-CoV-2). La gravedad de la pandemia ha provocado una escasez significativa de suministros médicos, entre ellos la hemodiálisis, sin embargo, el manejo de diálisis peritoneal en esta entidad por enfermedad por coronavirus 2019 (COVID-19) no ha recibido mucha atención, pudiendo ser una solución práctica para los pacientes con lesión renal aguda y enfermedad renal crónica en etapa terminal. Este artículo describimos nuestra experiencia con la implementación de diálisis peritoneal urgente para lesión renal aguda en pacientes con COVID-19.

Palabras clave: Enfermedad renal crónica. Lesión renal aguda. COVID-19. Diálisis peritoneal. Hemodiálisis.

\section{Peritoneal dialysis in the time of coronavirus disease 2019: Experience of five cases in a hospital of Mexico City}

\section{Abstract}

Acute kidney injury occurs in 4-23\% of cases in patients with SARS-CoV-2. The severity of the pandemic has caused a significant shortage of medical supplies, including hemodialysis, however, the management of peritoneal dialysis in this entity due to COVID-19 has not received much attention, and may be a practical solution for patients with injury acute kidney and end-stage chronic kidney disease. This article describes our experience with the implementation of urgent peritoneal dialysis for acute kidney injury in patients with COVID-19.

Key words: Chronic kidney disease. Acute kidney injury. COVID-19. Peritoneal dialysis. Hemodialysis. 


\section{Introducción}

Desde diciembre de 2019, el mundo se enfrenta a un nuevo desafío con un nuevo virus pandémico, el coronavirus 2 del síndrome respiratorio agudo severo (SARS-CoV-2), conocido como coronavirus 2019 y causante de la enfermedad por coronavirus 2019 (COVID-19). El sistema sanitario mundial se ha visto gravemente afectado debido al rápido aumento del número de pacientes. Los riñones no suelen ser el objetivo principal del SARS-CoV-2, pero sorprendentemente, la lesión renal aguda (LRA) ocurre en el 4 a $23 \%$ de los casos $^{1-5}$.

Estudios retrospectivos recientes han demostrado que los pacientes con COVID-19 que desarrollan LRA tienen un pronóstico extremadamente malo ${ }^{6-8}$. Para rescatar a estos pacientes severos, definitivamente se debe considerar la terapia de reemplazo renal (TRR). Sin embargo, en términos de control de infecciones y recursos médicos, es mucho más difícil realizar la hemodiálisis (HD) que antes de que ocurriera la pandemia ${ }^{9}$.

En países desarrollados como los EE.UU., la HD intermitente o la terapia de reemplazo renal continuo (TRRC) son los modos principales de TRR para el tratamiento de la $\operatorname{LRA}^{10}$. Sin embargo, en el pico de la enfermedad por coronavirus, la capacidad de diferentes centros médicos para proporcionar HD y TRRC para el tratamiento de LRA se ha visto abrumada debido al aumento en el número de pacientes con LRA que requieren de TRR combinado con escasez de personal relacionada con la enfermedad ${ }^{11}$.

Debido a esto, se han iniciado programas urgentes de diálisis peritoneal (DP). La DP se administra con mayor frecuencia como terapia de mantenimiento en pacientes con insuficiencia renal, pero también puede ser eficaz en pacientes con LRA en estadio $3^{12-15}$.

En un ensayo aleatorizado y controlado de pacientes con LRA en estadio 3 que recibieron DP o HD, el control metabólico, la mortalidad y las tasas de recuperación de la función renal fueron similares. Los metaanálisis tampoco mostraron diferencias entre la HD y la DP cuando se usaban para la LRA en los resultados clínicos, incluida la mortalidad, la recuperación renal y las complicaciones ${ }^{16,17}$.

Así, varios estudios han reportado un desempeño y resultados satisfactorios con $\mathrm{DP}^{18}$, requiriéndose esta modalidad en pacientes con COVID-19 y LRA hasta en el $9 \%$, donde la indicación para iniciar el reemplazo renal se maneja de acuerdo con las directrices internacionales ${ }^{19}$. En esta patología, el reemplazo renal es muy útil dada la gran cantidad de factores que son agresivos para el riñón, donde la HD ha sido la principal forma de terapia de reemplazo, pero la DP puede ser una alternativa segura con la colocación del catéter en la cabecera de los pacientes ${ }^{20}$.

La COVID-19 se ha aislado de diferentes muestras (muestras de sangre, hisopos orofaríngeos, orina, heces, etc.), pero no del efluente peritoneal; un estudio mostró en 1,070 muestras recolectadas de 205 pacientes con COVID-19 que las muestras de líquido de lavado broncoalveolar tuvieron tasas positivas más altas $(93 \%)$, seguidas de esputo (72\%), hisopos nasales (63\%), biopsia con cepillo de fibrobroncoscopio (46\%), hisopos faríngeos $(32 \%)$, heces $(29 \%)$ y sangre $(1 \%)^{21}$.

Este artículo describe nuestra experiencia de cinco casos con la implementación de DP urgente para LRA asociada a COVID- 19.

\section{Casos clínicos}

\section{Caso clínico 1}

Paciente de sexo masculino de 50 años de edad con antecedentes de enfermedad renal crónica de larga evolución en manejo con terapia de sustitución renal (HD), obesidad grado I, que inició padecimiento actual el 03.07.2020 con disnea de medianos esfuerzos, astenia, adinamia, tos seca y escalofríos motivo por el cual consulta al servicio de urgencias el día 04.07.2020, con signos vitales: tensión arterial (TA) 120/80 mmHg, frecuencia cardiaca (FC) $86 \mathrm{Ipm}$, frecuencia respiratoria (FR) $24 \mathrm{rpm}$, temperatura $37.1{ }^{\circ} \mathrm{C}$, saturación de oxígeno $\left(\mathrm{SaO}_{2}\right)$ del $86 \%$ al aire ambiente y del $96 \%$ con puntas nasales a $3 \mathrm{lpm}$. Se diagnosticó infección por SARS-CoV-2 mediante prueba de reacción en cadena de la polimerasa (RT-PCR) y tomografía de tórax. El paciente presentó hiperpotasemia más acidosis metabólica refractarias a tratamiento, por lo que se indicó colocación de catéter Mahurkar para realización de tres sesiones de HD, posteriormente se realizó la colocación de catéter Tenckhoff sin complicaciones, se realizaron 28 recambios con solución dializante al 1.5\%, con un balance final negativo de $650 \mathrm{ml}$, dado de alta a los 11 días de estancia hospitalaria. A su egreso se citó a los 21 días para seguimiento encontrándose con catéter funcional.

\section{Caso clínico 2}

Paciente de sexo masculino de 56 años con antecedente de diabetes tipo 2 (DT2) e hipertensión arterial sistémica (HAS) de 16 años de evolución sin 
tratamiento ni seguimiento durante los últimos dos años, acudió a valoración por odinofagia, astenia, adinamia, así como evacuaciones diarreicas. Se ingresó con diagnóstico de neumonía por SARS-CoV-2 corroborado por RT-PCR y tomografía de tórax, así como enfermedad renal crónica agudizada presentando acidosis metabólica refractaria, sobrecarga hídrica y uremia, por lo que se colocó catéter Mahurkar con el que recibió cuatro sesiones de HD con posterior colocación de catéter Tenckhoff para diálisis peritoneal.

Durante su estancia presentó infarto de miocardio sin elevación persistente del segmento ST, corroborado posteriormente por ecocardiograma transtorácico 2D, para el cual recibió manejo antiisquémico. Se realizaron 42 recambios con solución dializante al $2.5 \%$, hasta su egreso con un balance final negativo de $6,550 \mathrm{cc}$, dado de alta a los 25 días de estancia hospitalaria. A su egreso se citó a los 24 días para seguimiento encontrándose con catéter funcional.

\section{Caso clínico 3}

Paciente de sexo femenino de 58 años con antecedente de DT2 de larga evolución, hipotiroidismo primario e HAS de un año de diagnóstico, así como enfermedad renal crónica de tres años de evolución en tratamiento con diurético de asa de manera intermitente. Acudió por presentar astenia, adinamia, malestar general, disnea progresiva a ortopnea. A su llegada TA 70/60 mmHg, FC 140 lpm, FR 49 rpm y SO $27 \%$. Requirió manejo avanzado de la vía aérea y por acidosis metabólica refractaria, así como datos de uremia, por lo que se colocó catéter Mahurkar. Requirió manejo en unidad de cuidados intensivos durante 32 días, dada de alta a los 40 días de estancia hospitalaria. Durante su estancia recibió tres sesiones de HD, con posterior colocación de catéter Tenkchoff con el cual recibió 21 recambios con 1,000 cc de solución dializante al 1.5\% con un balance total final de $-1,200 \mathrm{cc}$. A su egreso se citó a los 30 días para seguimiento encontrándose con catéter funcional.

\section{Caso clínico 4}

Hombre de 44 años de edad con antecedente de DT2 e hipertensión arterial de 15 años de evolución. Inició padecimiento actual en junio 2020 por edema de miembros inferiores progresivo, por lo que acudió al servicio de urgencias el 11.07.20 con los siguientes signos vitales: TA $85 / 54 \mathrm{mmHg}$, FC $36 \mathrm{lpm}$, FR
$22 \mathrm{rpm}$, temperatura $36.6{ }^{\circ} \mathrm{C}$ y $\mathrm{SaO}_{2} 93 \%$ con puntas nasales $2 \mathrm{lpm}$. Se realizó RT-PCR y tomografía de tórax, compatibles con infección por SARS-CoV-2. A su ingreso presentó hiperpotasemia y acidosis metabólica refractarias a tratamiento, uremia y sobrecarga hídrica, por lo que se realizó colocación de catéter Mahurkar para realización de tres sesiones de HD. Durante su estancia hospitalaria se realizó colocación de catéter Tenckhoff sin complicaciones, con realización de 28 recambios con 1,500 cc de solución dializante al $2.5 \%$ con un balance total final de $-3,260 \mathrm{cc}$. Durante su hospitalización posterior a la colocación del catéter Tenckhoff fue colocado en posición prono para el tratamiento de la hipoxemia durante tres días. Fue egresado a los 11 días de hospitalización. Se citó a los 30 días para seguimiento, encontrándose con catéter funcional.

\section{Caso clínico 5}

Mujer de 70 años de edad con antecedente de DT2, HAS desde hace 15 años y enfermedad renal crónica que inició padecimiento actual el 02.07.2020 por tos no productiva, fiebre, cefalea, mialgias, artralgias y disnea de medianos esfuerzos, por lo que acudió al servicio de urgencias el 10.07.20 por exacerbación de disnea con los siguientes signos vitales: TA 98/60 mmHg, FC 107 lpm, FR 23 rpm, temperatura $37.2{ }^{\circ} \mathrm{C}$ y SaO $292 \%$ con mascarilla facial a $5 \mathrm{I} /$ minuto. Se realizó RT-PCR y tomografía de tórax compatibles con infección por SARS-CoV- 2. Cinco días posteriores a su ingreso, la paciente presentó hiperpotasemia refractaria a tratamiento y uremia, por lo que se decidió colocación de catéter Mahurkar, con requerimiento de tres sesiones de HD. Se realizó colocación de catéter Tenckhoff sin complicaciones con realización de sesiones con 1,500 cc de solución dializante al 1.5\% con estancia de cuatro horas en cavidad con dos recambios diarios con un balance total final de $-250 \mathrm{cc}$. Durante su hospitalización posterior a la colocación del catéter Tenckhoff fue colocada en posición prono para el tratamiento de la hipoxemia durante cinco días. Se citó a los 30 días para seguimiento, encontrándose con catéter funcional.

\section{Discusión}

Durante el 2020, en el Departamento de Medicina Interna del Hospital Gea, un centro de tercer nivel de atención de la Ciudad de México, reportaron 239 pacientes con diagnóstico de infección por SARS-CoV-2 
mediante test RT-PCR y tomografía de tórax, dentro de los cuales un total de 79 pacientes desarrollaron LRA, de estos, 15 pacientes (18.9\%) requirieron TRR, todos ellos recibieron HD inicialmente, sin embargo debido a la gran demanda de esta terapia y la escasez de personal y suministros, comenzamos un programa de DP, por lo que cinco pacientes fueron cambiados a esta modalidad, tres pacientes ya tenían el diagnóstico de enfermedad renal crónica y dos pacientes fueron diagnosticados a su ingreso hospitalario, durante su estancia una paciente requirió de ventilación mecánica invasiva y el resto se encontraron con dispositivos como puntas nasales y mascarilla reservorio, dos de los cuatro pacientes no intubados fueron colocados en posición prono para el tratamiento de la hipoxemia, sin presentar ninguna alteración en el sitio de inserción del catéter, ningún paciente presentó alguna complicación asociada a la DP como infecciones o disfunción y ninguno requirió suplementación intermitente con HD. El tiempo promedio de estancia hospitalaria fue de 22 días y el número de sesiones promedio de HD previo al cambio de DP fue de tres sesiones, la colocación de los catéteres fue con la técnica quirúrgica rectovesical, sin presentar complicaciones durante el transquirúrgico. La dosis prescrita para los pacientes que recibieron DP después de las 48 a 72 horas iniciales posteriores a su colocación, fue un volumen de llenado de 1 a 1.5 litros, 3 a 4 intercambios por día, con un tiempo de permanencia de 3 a 4 horas y balance total promedio de $-2,250$ cc en 7 días (Tabla 1).

Los cinco pacientes fueron dados de alta a su hogar, egresando con el catéter Tenkchoff de forma permanente y con un desenlace favorable, todos fueron citados un mes posterior a su egreso como parte de su control y seguimiento, encontrándose clínicamente estables y con adecuada funcionalidad del catéter.

El uso de la DP en este escenario tiene importantes ventajas, primero, no hay necesidad de acceso vascular, ya que los pacientes con niveles elevados de dímero $D$ y productos de degradación de fibrina, como se observa en la COVID-19, tienen más probabilidades de presentar coagulación intravascular diseminada ${ }^{22,23}$, por tanto debido a la sepsis grave y la coagulopatía concomitante asociada con COVID-19, se prefiere evitar el acceso vascular cuando sea posible. En segundo lugar, el costo general de la DP es significativamente menor que el de la $\mathrm{HD}$, que requiere maquinaria y suministros más caros. En tercer lugar, se puede educar al personal sobre cómo realizar los recambios de DP de manera segura con pocos recursos y en poco

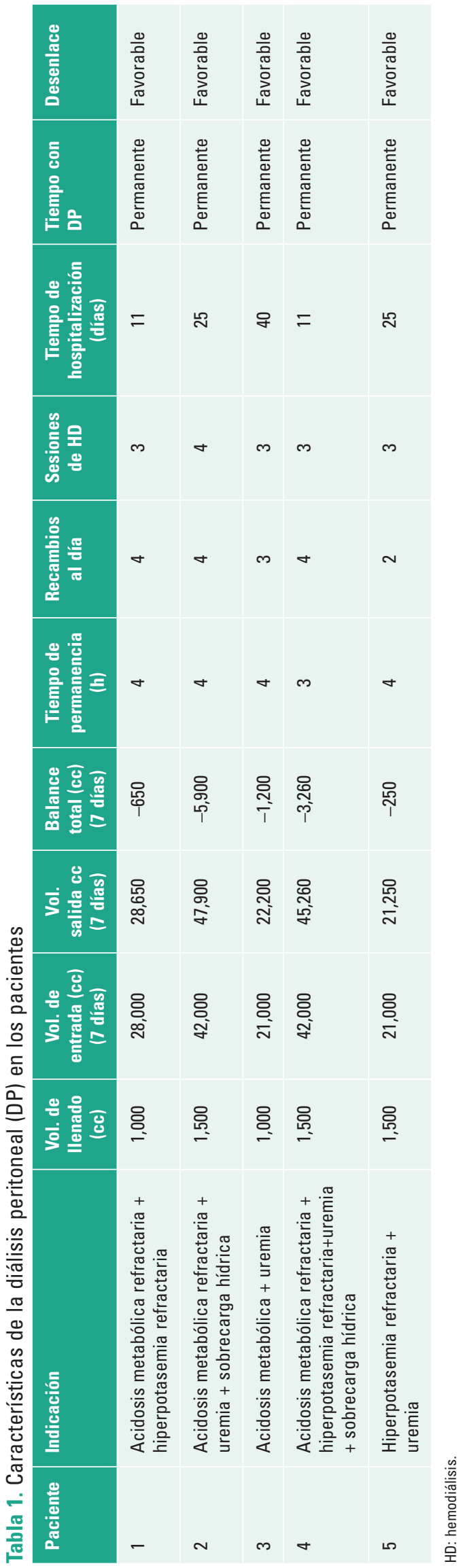


tiempo ${ }^{11}$. El entrenamiento de DP es menos desafiante técnicamente en comparación con el entrenamiento en HD intermitente $y$, por lo tanto, es una opción viable para el entrenamiento de enfermeras y técnicos durante una pandemia ${ }^{24}$. Finalmente, a diferencia de la HD intermitente, la DP no requiere una conexión de suministro de agua de dializado, que podría representar un factor limitante en varias ubicaciones temporales de unidades de cuidados intensivos construidas en respuesta al aumento de COVID-1911.

Ahora, en los pacientes que ya tienen esta modalidad de diálisis de forma permanente, en estas circunstancias, una ventaja es que pueden llevar a cabo el tratamiento de diálisis en sus propios hogares, ya que de esta manera el paciente con DP puede minimizar el contacto interpersonal al evitar la necesidad de llegar al centro de HD tres veces por semana. Además, el paciente anula la exposición al virus mientras viaja desde su casa al centro en los servicios de transporte público y espera el inicio del tratamiento de diálisis. De igual manera, los pacientes con DP tienen la ventaja de reducir el contacto cercano con los trabajadores de la salud, que pueden verse potencialmente afectados por la infección por SARS-CoV-225.

\section{Conclusión}

En conclusión, la DP representa una terapia alternativa factible a la HD en escenarios con alta demanda de esta y escasez crítica de recursos, es aplicable a diversas situaciones de tratamiento para pacientes graves con COVID-19, coincidiendo con las necesidades sociales y técnicas existentes y exacerbadas durante esta pandemia, aunado a la actual crisis global. Constituye una opción para hacer frente al aumento del número de pacientes infectados en todo el mundo y más aún en países en desarrollo que no cuentan con el personal y suministros necesarios para ofrecer este tipo de terapias. Por lo tanto, en este contexto, la DP representa la mejor TRR para pacientes con enfermedad renal en etapa terminal durante el brote de COVID-19, como terapia inicial y de mantenimiento.

\section{Agradecimientos}

Los autores agradecen a la División de Medicina Interna del Hospital General Dr. Manuel Gea González, Ciudad de México, México.

\section{Financiamiento}

La presente investigación no ha recibido ayudas específicas provenientes de agencias del sector público, sector comercial o entidades sin ánimo de lucro.

\section{Conflicto de intereses}

No existe conflicto de intereses por parte del autor y coautores de este artículo.

\section{Responsabilidades éticas}

Protección de personas y animales. Los autores declaran que para esta investigación no se han realizado experimentos en seres humanos ni en animales.

Confidencialidad de los datos. Los autores declaran que han seguido los protocolos de su centro de trabajo sobre la publicación de datos de pacientes.

Derecho a la privacidad y consentimiento informado. Los autores han obtenido el consentimiento informado de los pacientes y/o sujetos referidos en el artículo. Este documento obra en poder del autor de correspondencia.

\section{Bibliografía}

1. Wu Z, McGoogan JM. Characteristics of and important lessons from the coronavirus disease 2019 (COVID-19) outbreak in China: summary of a report of 72314 cases from the Chinese Center for Disease Control and Prevention. JAMA. 2020;323:1239.

2. Guan WJ, Ni ZY, Hu Y, Liang WH, Ou CQ, He JX, et al. Clinical characteristics of coronavirus disease 2019 in China. N Engl J Med. 2020;382:1708-20.

3. Arentz M, Yim E, Klaff L, Lokhandwala S, Riedo FX, Chong M, et al. Characteristics and outcomes of 21 critically ill patients with COVID-19 in Washington State. JAMA. 2020;323:1612

4. Shi Q, Zhao K, Yu J, Liu Z, Shang L, Zhou F, et al. Clinical characteristics of 101 non surviving hospitalized patients with COVID-19: a single center, retrospective study. Front Med. 2020;14(5):601-12.

5. Ronco C, Navalesi P, Vincent JL. Coronavirus epidemic: preparing for extracorporeal organ support in intensive care. Lancet Respir Med. 2020;8:240-1.

6. Chen T, Wu D, Chen H, Yan W, Yang D, Chen G, et al. Clinical characteristics of 113 deceased patients with coronavirus disease 2019: retrospective study. BMJ. 2020;368:m1091.

7. Cheng Y, Luo R, Wang K, Zhang M, Wang Z, Dong L,et al. Kidney disease is associated with in-hospital death of patients with COVID-19. Kidney Int. 2020;97(5):829-38.

8. Deng Y, Liu W, Liu K, Fang YY, Shang J, Zhou L, et al. Clinical characteristics of fatal and recovered cases of coronavirus disease 2019 (COVID-19) in Wuhan, China: a retrospective study. Chin Med J (Engl). 2020;133(11):1261-7.

9. Nagatomo M, Yamada H, Shinozuka K, Shimoto M, Yunoki T, Ohtsuru S. Peritoneal dialysis for COVID-19-associated acute kidney injury. Crit Care. 2020;24:309.

10. Ronco C, Zanella M, Brendolan A, Milan M, Canato G, Zamperetti N, et al. Management of severe acute renal failure in critically ill patients: an international survey in 345 centres. Nephrol Dial Transplant. 2001;16(2):230-7.

11. Sourial MY, Sourial MH, Dalsan R, Graham J, Ross M, Chen W, et al. Urgent peritoneal dialysis in patients with COVID-19 and acute kidney injury: A single-center experience in a time of crisis in the United States. Am J Kidney Dis. 2020;76(3):401-6.

12. Cullis $B$, Abdelraheem M, Abrahams G, Balbi A, Cruz DN, Frishberg $Y$, et al. Peritoneal dialysis for acute kidney injury. Perit Dial Int. 2014; 34(5):494-517. 
13. Gabriel D, Nascimento G, Caramori J, Martim LC, Barretti P, Balbi AL. Peritoneal dialysis in acute renal failure. Ren Fail. 2006;28(6):451-6.

14. Gabriel D, Nascimento G, Caramori J, Martim LC, Barretti P, Balbi AL. High volume peritoneal dialysis for acute renal failure. Perit Dial Int. 2007;27(3): 277-82

15. Gabriel D, Caramori J, Martim L, Barretti P, Balbi AL.High volume peritoneal dialysis vs daily hemodialysis: a randomized, controlled trial in patients with acute kidney injury. Kidney Int Suppl. 2008;73(108):S87-S93.

16. Chionh C, Soni S, Finkelstein F, Ronco C, Cruz DN. Use of peritoneal dialysis in AKI: a systematic review. Clin J Am Soc Nephrol. 2013;8(10):1649-60

17. Liu L, Zhang L, Liu G, Fu P. Peritoneal dialysis for acute kidney injury. Cochrane Database Syst Rev. 2017;12:CD011457.

18. Ronco C, Bellomo R, Kellum JA. Acute kidney injury. Lancet. 2019; 394(10212):1949-64.

19. Chávez Valencia V, Lagunas Rangel FA, Orizaga de la Cruz C. Letter to the Editor: Safety and efficacy of bedside peritoneal dialysis catheter placement in the COVID-19 Era: Initial experience at a New York City Hospital. World J Surg. 2020;44(10):3584-5.
20. Chen N, Zhou M, Dong X, Qu J, Gong F, Han Y, et al. Epidemiological and clinical characteristics of 99 cases of 2019 novel coronavirus pneumonia in Wuhan, China: a descriptive study. Lancet. 2020; 395(10223):507-13.

21. Wang W, Xu Y, Gao R, Lu R, Han K, Wu G, et al. Detection of SARS-CoV-2 in different types of clinical specimens. JAMA. 2020;323(18):1843-4.

22. Tang $\mathrm{N}$, Li D, Wang $\mathrm{X}$, Sun $\mathrm{Z}$. Abnormal coagulation parameters are associated with poor prognosis in patients with novel coronavirus pneumonia. J Thromb Haemost. 2020;18(4):844-7.

23. Thachil J, Tang N, Gando S, Falanga A, Cattaneo M, Levi M, et al. ISTH interim guidance on recognition and management of coagulopathy in COVID-19. J Thromb Haemost. 2020;18(5):1023-6.

24. El Shamy O, Sharma S, Winston J, Uribarri J. Peritoneal dialysis during the coronavirus disease-2019 (COVID-19) pandemic: acute inpatient and maintenance outpatient experiences. Kidney Med. 2020;2(4):377-80.

25. Ran L, Chen X, Wang Y, et al. Risk factors of healthcare workers with corona virus disease 2019: A retrospective cohort study in a designated hospital of Wuhan in China. Clin Infect Dis. 2020;71(16):2218-21. 